\title{
On the effect of the background wind on the evolution of interplanetary shock waves
}

\author{
C. Jacobs, S. Poedts, B. Van der Holst, and E. Chané
}

\author{
Centre for Plasma Astrophysics, K.U. Leuven, Celestijnenlaan 200B, 3001, Leuven, Belgium \\ e-mail: Carla.Jacobs@wis.kuleuven.ac.be
}

Received 16 July 2004 / Accepted 20 September 2004

\begin{abstract}
The propagating shock waves in the solar corona and interplanetary (IP) space caused by fast Coronal Mass Ejections (CMEs) are simulated numerically and their structure and evolution is studied in the framework of ideal magnetohydrodynamics (MHD). Due to the presence of three characteristic velocities and the anisotropy induced by the magnetic field, the CME shocks generated in the lower corona can have a complex structure and topology including secondary shock fronts, over-compressive and compound shocks, etc. The evolution of these CME shocks is followed during their propagation in IP space up to $r=30 R_{\odot}$. Here, particular attention is given to the effect of the background solar wind on the evolution parameters of the fast CME shocks, i.e. shock speed, deformation of the leading shock front and the CME plasma, stand-off distance of the leading shock front, direction, spread angle, etc. First, different "frequently used" solar wind models are reconstructed with the same numerical code, the same numerical technique on exactly the same numerical grid (and thus the same numerical dissipation), the same boundary conditions, and the same normalization. Then, a simple CME model is superposed on three different solar wind models, again using exactly the same initial conditions. The result is a fair comparison and thus an objective study of the effect of the background wind on the CME shock evolution. This effect is surprisingly substantial and can be quantified due to the uniformity of the normalization of the used models and simulation techniques.
\end{abstract}

Key words. solar wind - Sun: coronal mass ejections (CMEs)

\section{Motivation and key role of CME shocks}

Coronal Mass Ejections (CMEs) play a crucial role in space weather and a careful study of the origin, the structure, and the propagation characteristics of these violent solar phenomena is essential for a deeper insight into space weather physics. The fast CMEs owe their importance to the propagating shock waves they generate. The shape and structure of these MHD shocks depend critically on their velocity and on the magnetic field, the pressure, etc. of the background plasma. Steinolfson \& Hundhausen (1990) predicted that, when these shocks are in the so-called switch-on regime, the shock front must be dimpled in order to avoid a discontinuity at the top. Later, De Sterck \& Poedts (1999) showed that, in the case of a "magnetically dominated" upstream plasma, such a dimpled (steady) shock front is indeed found in numerical simulations, together with several secondary shock fronts. Moreover, the resulting shock topology turned out to be rather complex involving almost all different MHD shock types simultaneously, including fast shocks, fast switch-on shocks, intermediate shocks, double compound shocks, slow shocks, hydrodynamic shocks, tangential discontinuities, etc. As the topology of these MHD shocks depends critically on the parameter values, e.g. the velocity of the CME itself and the plasma beta of the background wind, this complexity may help to derive some of the values of these parameters provided these shocks can be observed in detail in the low corona. In the mean time, Terasawa et al. (2002) carefully investigated the very large solar event of July 14, 2000 (Bastille day event). Their multi-satellite triangulation of data from 5 different spacecraft yielded the 3D geometry of the interplanetary (IP) shock generated by this event. It turned out that the shock front is indeed dimpled, which is a clear magnetic (MHD) effect.

There is another reason why CME shocks are important for space physics: the shocks generated by CMEs can be observed in the IP medium since they accelerate particles and these accelerated particles emit radio waves (see e.g. van der Holst et al. 2002a, and references therein). The frequency drift of the resulting type-II radio emissions are related to the dynamics of the shock (and the related CME) from the high corona into the interplanetary medium. Changes in the shock and CME dynamics can be caused by interaction with structures in the interplanetary space, e.g. collision with another CME. According to Burlaga et al. (2002) two thirds of the interplanetary ejecta are complex, i.e. last several days and may consist of two or more CMEs colliding together. These collisions can lead to shockdense matter interaction (dense core of an ejected filament) or shock-shock interaction (Gopalswamy et al. 2001).

Clearly, the theoretical modeling of the evolution of CMEs can be divided into different sub-problems. Such a 
sub-problem, e.g., is the observational study and modeling of the fast and slow "quiet" solar wind where questions regarding the heating source(s) and acceleration mechanism(s), the required amount of energy, and the location of the acceleration and heating sources of the fast wind component need to be answered. Another sub-problem is the initiation of CMEs: why do CMEs occur and how are they triggered? Next, there is the sub-problem of the propagation of CMEs and, in particular, the observed time-height curves need to be explained. Also the evolution of the structure of the CMEs and the leading shock fronts during their propagation through the interplanetary medium needs to be studied. The modification(s) of the MHD shock structure may contain important clues to understanding the propagation properties of CMEs. The impact of CMEs or magnetic clouds on the Earth's magnetosphere is another crucial sub-problem in which the MHD shock complexity is important. The interaction of the CME leading shock front with the bow shock at the Earth's magnetosphere drastically affects the reconnection characteristics of the magnetic field lines. Clearly, this affects the "geo-effectiveness" of the magnetic storms. In the present paper, we concentrate on the first and the third sub-problem mentioned above: the modeling of the background wind and its effect on the evolution properties of the CME shocks.

In the next section, three solar wind models that are often used in the literature are reconstructed. These reconstructions are obtained with the same numerical code so that the only difference in the models lies in the physics included in the equations. This is important for an objective comparison. In Sect. 3, we first discuss different CME initiation models used in the literature. We then describe the simple model used in this initial study of the evolution of CME shocks in the solar wind. We concentrate on fast CMEs and consider two different launch angles. In spite of its simplicity our CME model has some remarkable generic features. Section 4 contains the results of these CME evolution studies. The effects of the background wind model on the evolution parameters of the IP CMEs are quantified. Next, in Sect. 5, we focus on the magnetic reconnection and the back flows in the current sheet that forms behind the CMEs when they are launched in the equatorial region. We end with some discussion and conclusions in Sect. 6.

\section{Modeling the (pre-event) solar wind}

\subsection{Axi-symmetric (2.5D) wind models}

Pneumann \& Kopp (1971) constructed the first twodimensional (axi-symmetric) MHD model for the solar corona by solving directly the steady state equations. This model included both a helmet streamer and open field regions. Sakurai (1985, 1990) derived an analytical 2D generalization of the Weber-Davis wind model (Weber \& Davis 1967). Most axisymmetric wind models, however, are numerical solutions obtained by integrating the time-dependent MHD equations by using a time-asymptotic approach. The energy equation is often simplified, e.g. by considering a polytropic relation between pressure and density, sometimes even isothermal. Such simplified polytropic models yield surprisingly good approximations and can reproduce many qualitative features of the observed solar corona. But the plasma density and temperature in these models are not in quantitative agreement with the observations. Therefore, more recent models focus on improving the energy equation, e.g. by including source terms to model the effect of additional heating or acceleration. Below, we briefly discuss some of these models that have been used to simulate the evolution of IP CMEs and, in particular, the MHD shocks they generate.

Keppens \& Goedbloed $(1999,2000)$ derived a 2.5D, ideal MHD solution starting from $1.25 R_{\odot}$. This polytropic model (with $\gamma=1.13$ ) includes differential rotation and both a "wind" and a "dead" zone to produce a high speed wind from polar coronal holes and a low speed wind above equatorial streamers. The dead zone is obtained by imposing an initial dipole field and keeping the poloidal component of the velocity field zero between latitudes $\pm 30^{\circ}$. These authors also impose a mass flux $f_{\text {mass }}=0.01377$ in the "wind" zone, while in the "dead" zone $f_{\text {mass }}=0$. This wind model has been used to study the effect of the "dead" zone and the magnetic field strength on stellar wind properties and also to study CME evolution (van der Holst et al. 2002b; Poedts et al. 2003).

Wang et al. $(1995,1998)$ constructed a 2D, polytropic wind solution including both heating and momentum source terms to obtain mass fluxes similar to those observed in the solar wind. By combining both constant and latitude-dependent boundary conditions with different values of the polytropic index, these authors showed that the background wind has a drastic effect on the evolution properties of CMEs superposed on it. Later, Suess et al. (1999) upgraded this wind model to a two-fluid MHD model of the global structure of the solar corona which contains energy exchange between electrons and protons due to collisions. It still includes a momentum source term and the volumetric heat source is different for protons and electrons so that a high $T_{\mathrm{p}} / T_{\mathrm{e}}$ ratio is obtained above the polar coronal holes and a low $T_{\mathrm{p}} / T_{\mathrm{e}}$ ratio above the equatorial streamer.

\subsection{Reconstruction of three wind models}

Wang et al. (1995) showed with a parameter study that the preevent corona is a crucial factor in dictating CME properties. In this parameter study only a polytropic wind was considered and only the polytropic index was varied. Yet, this parameter turned out to have a substantial influence on the CME evolution. Here, we extend the parameter study of Wang et al. (1995) by considering three different $2.5 \mathrm{D}$, axi-symmetric wind models that have been used in the literature for CME evolution studies.

The three stationary MHD 2.5D wind solutions used in the present parameter study are all parameterized in the same way, viz. by choosing at the reference position $r_{*}=1.01 R_{\odot}$ the temperature $T_{*} \approx 1.5 \times 10^{6} \mathrm{~K}$, number density $N_{*} \approx 10^{8} \mathrm{~cm}^{-3}$, angular velocity $\Omega_{*} \approx 2.8 \times 10^{-6} \mathrm{rad} / \mathrm{s}$, and the radial component of the dipole magnetic field with $B_{r *} \approx 2.2 \mathrm{G}$ at the pole. The three wind models are normalized with respect to the density $\rho$, speed of sound $c$ (both taken at the reference position) and $r_{*}$. Far away from the sun the plasma outflow is superfast and continuous. The three wind models are also computed 
Table 1. Wind characteristics at $30 R_{\odot}$.

\begin{tabular}{llll}
\hline \hline & Model 1 & Model 2 & Model 3 \\
\hline Density $\left[\mathrm{m}^{-3}\right]$ & & & \\
$\quad$ Pole & $6.1 \times 10^{8}$ & $1.46 \times 10^{9}$ & $3.05 \times 10^{9}$ \\
$\quad$ Equator & $7.4 \times 10^{8}$ & $2.93 \times 10^{9}$ & $2.86 \times 10^{9}$ \\
$\quad$ Ratio & 0.82 & 0.50 & 1.07 \\
$\quad$ Velocity $\left[\mathrm{km} \mathrm{s}^{-1}\right]$ & & & \\
$\quad$ Pole & 330 & 720 & 685 \\
$\quad$ Equator & 295 & 335 & 370 \\
$\quad$ Ratio & 1.12 & 2.15 & 1.85 \\
Temperature $[\mathrm{K}]$ & & & \\
$\quad$ Pole & $0.82 \times 10^{6}$ & $1.18 \times 10^{6}$ & $0.89 \times 10^{6}$ \\
$\quad$ Equator & $0.83 \times 10^{6}$ & $0.36 \times 10^{6}$ & $0.89 \times 10^{6}$ \\
$\quad$ Ratio & 0.99 & 3.28 & 1.00 \\
Total mass & & & \\
flux $\left[M_{\odot} / \mathrm{yr}\right]$ & $0.30 \times 10^{-13}$ & $1.54 \times 10^{-13}$ & $2.24 \times 10^{-13}$ \\
\hline
\end{tabular}

on the same $301 \times 90$ non-equidistant, accumulated grid that ranges from 1 to $30 R_{\odot}$. The grid accumulation is specified by $\delta r_{\max } / \delta r_{\min }=180, \delta \theta_{\max } / \delta \theta_{\min }=5$. We also used the same numerical code: all simulations discussed in the present paper are obtained with the finite volume code VAC (Versatile Advection Code, Tóth 1996) using the Lax-Friedrichs upwind solver. In contrast to Keppens \& Goedbloed (1999, 2000) we did not prescribe a dead zone but we did use the same differential rotation as these authors for all three winds, viz. $\Omega(\theta)=\Omega_{0}+\Omega_{2} \cos ^{2} \theta+\Omega_{4} \cos ^{4} \theta$, where this $\Omega(\theta)$ profile and the constants $\Omega_{0}, \Omega_{2}$, and $\Omega_{4}$ are chosen such in order to fit the observations. Last but not least, we use the same boundary conditions in these different wind solutions. At $r=1$ we fixed the density $\rho$, the temperature $T$ and the angular velocity $\Omega$. The radial magnetic flux $r^{2} B_{r}$ of the above mentioned dipole field is also kept fixed at the solar surface. We set the perpendicular component of the poloidal velocity field equal to zero. In fact, we extrapolate $r^{2} \rho v_{r}$ at the boundary and then determine $v_{\theta}$, such that the poloidal velocity component perpendicular to the poloidal magnetic field vanishes. Hence, the only variables left free at the boundary are the parallel component of the poloidal velocity field and the $\theta$ - and $\varphi$-component of the magnetic field.

The three different wind models thus differ only by the physics included in the terms of the model equations and in source terms. Solar wind model 1 is the standard polytropic wind model with polytropic index $\gamma=1.05$. This wind model is fairly popular in the literature in spite of its remarkable shortcomings. It has e.g. a too small ratio of the polar versus the equatorial wind velocity (see Table1). Wind model 2 is a reconstruction of the wind model used by the Michigan group of T. Gombosi (see e.g. Groth et al. 2000). It is obtained by means of a heating source term which in normalized form is given by:

$Q=\rho q_{0} \mathrm{e}^{-\frac{(r-1)^{2}}{\sigma^{2}}}\left(T_{0}-\gamma \frac{p}{\rho}\right)$,

very similar to the volumetric heating function used by Groth et al. (2000). With the value $q_{0}=25$ and the profiles of $T_{0}$ and $\sigma$ having a $\theta$ dependence such that $T_{0}=1.0$ at the equator, $T_{0}=1.75$ at the pole, $\sigma=4.5$ at the equator and $\sigma=9$ at the pole. Compared to Groth et al. (2000) the transition of these parameters from pole to equator is somewhat smoother. This heating source term yields a much better wind model than the polytropic wind, with the right velocity ratio. Wind model 3 is again a polytropic model but now including an additional pressure gradient due to the Alfvén waves (Hollweg 1973; Jacques 1977). The extra Alfvén wave pressure, $p_{w}$, in the momentum equation, is determined by:

$$
\frac{\partial \epsilon_{ \pm}}{\partial t}+\nabla \cdot\left[\left(\boldsymbol{v} \pm \boldsymbol{v}_{A}\right) \epsilon_{ \pm}\right]=-\frac{1}{2} \epsilon_{ \pm} \nabla \cdot \boldsymbol{v},
$$

where $\epsilon_{ \pm}$is the average Alfvén wave density for the parallel and anti-parallel propagating waves and $\boldsymbol{v}_{A}$ is the Alfvén velocity. The extra Alfvén wave pressure is given by

$p_{w}=\frac{\epsilon_{+}+\epsilon_{-}}{2}$.

This wind model also has the "right" velocity ratio and has, compared to wind model 2, a smoother density transition going from the equator towards the poles.

The three wind models are illustrated in Fig. 1 showing the density (gray scale), velocity vectors, the magnetic field lines, the direction dependent Alfvénic, slow, and fast magnetosonic points, and the corresponding total Angular Momentum Fluxes (AMF)

$\mathrm{AMF}=2 \pi \int_{0}^{\pi} r^{3} \sin ^{2} \theta\left(\rho v_{r} v_{\phi}-B_{r} B_{\phi}\right) \mathrm{d} \theta$,

for all three winds. Near the solar base the AMF is almost entirely due to the magnetic tension. Away from the sun, the contribution of the AMF due to advection differs significantly for the different wind models. The sum of both contributions should be independent of the radius for steady state solutions. For clearness of the figure, the plotting domain in Fig. 1 is restricted to $15 R_{\odot}$. To have a better notion of the differences between the three winds, Fig. 2 shows the variation of density and radial velocity on a constant radius of $30 R_{\odot}$. Table 1 gives an overview of the polar and equatorial wind density, velocity and temperature at $30 R_{\odot}$ and of the total mass flux for the three different models.

\section{CME initiation models}

\subsection{Short review}

Klimchuk (2000) reviewed the different theoretical models for CME initiation and distinguished two types of models, viz. directly driven models and storage and release models. He concluded that all these models have difficulty explaining one or more aspects of the observations. Z. Mikić (private communication, 2001) also concluded that the present CME models are too idealized: the geometry is not realistic, there is no fine structure, the models are too dissipative, not fully selfconsistent, etc. Hence, a lot of work remains to be done and the present models need to be improved: $3 \mathrm{D}$ extensions need to be created including fine structure, real (instead of numerical) dissipation, a realistic shearing of foot points, etc.

In summary, CME shock evolution studies very often apply oversimplified generation models for the CMEs. 

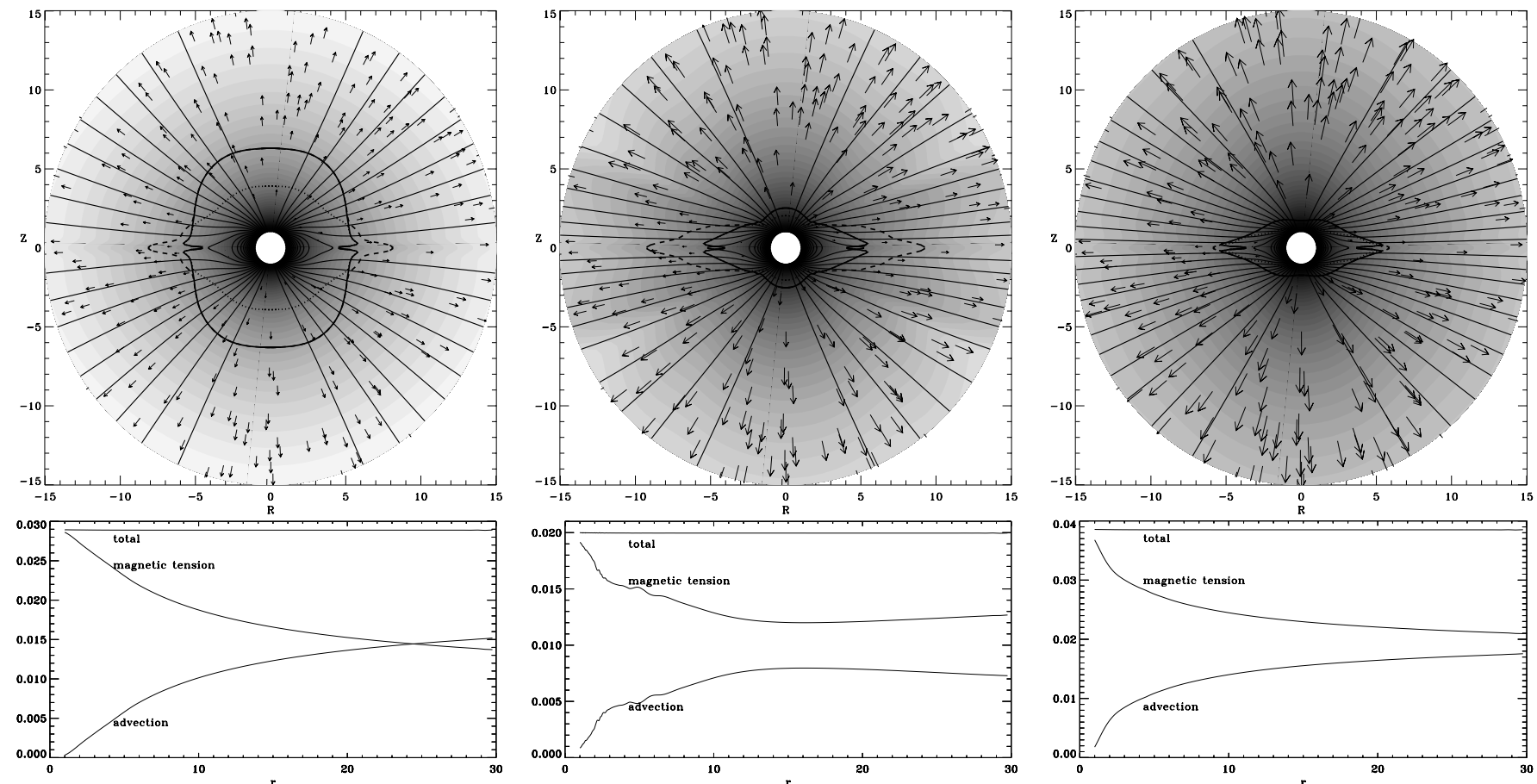

Fig. 1. The three steady wind solutions (upper row) and the corresponding total angular momentum fluxes (lower row). Left: the (standard) polytropic model with $\gamma=1.05$; middle: MHD wind model with energy source term (cf. Groth et al. 2000); right: polytropic MHD wind model with Alfvén wave heating term. The gray scale represents the density (log-scale), black lines are the magnetic field lines, and arrows denote the radial velocity. Also shown on these plots are the direction dependent Alfvénic (solid line), slow (dotted line), and fast (dashed line) magnetosonic points.
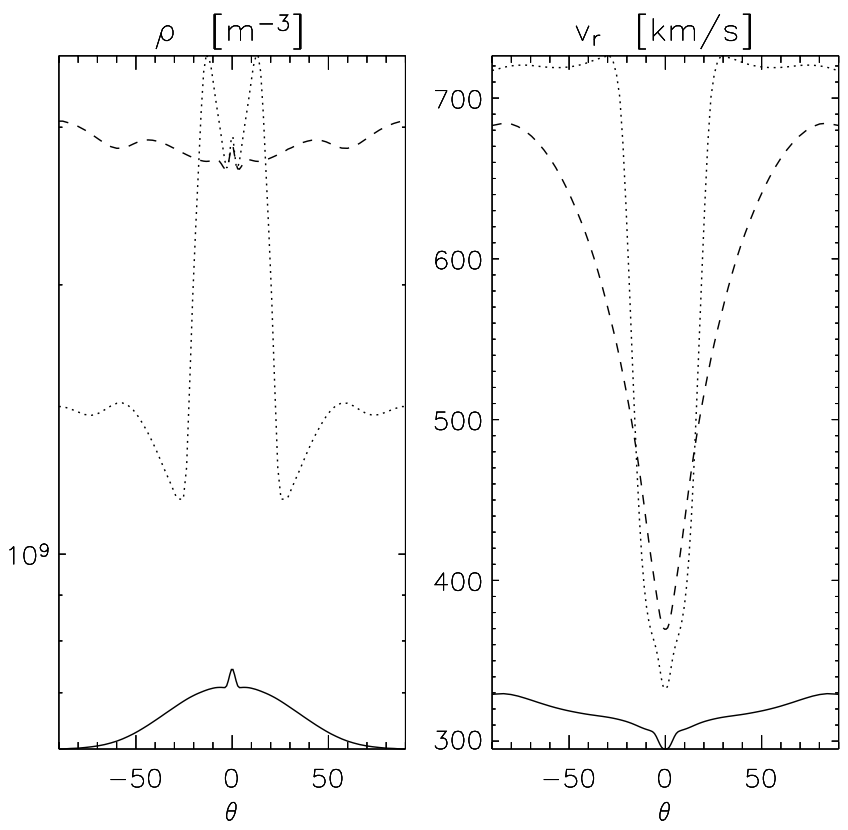

Fig. 2. The density (left) and radial velocity (right) profile for the three wind models at $30 R_{\odot}$. Solid line: model 1 , dotted line: model 2 , dashed line: model 3. The $x$-axis indicates the latitude $\theta\left(\theta=0^{\circ}\right.$ is the equator).

Groth et al. (2000) used a "density-driven" model which simply means that a high-density (and high-pressure) plasma blob is superposed on the solar wind. Other simplified models generate a pressure pulse with or without an additional velocity change (i.e. a kind of "nozzle" boundary condition), see e.g. Wang et al. (1995); Odstrčil \& Pizzo (1999). Keppens \& Goedbloed (2000) on the other hand impose an extra mass flow to generate the CMEs.

More realistic CME evolution simulations make use of (3D) analytic CME initiation models (such as the models of the self-similar Gibson \& Low (1998) family (Gombosi et al. 2000) or the Titov \& Démoulin (1999) model (Roussev et al. 2003)), or simulate the evolution of reconstructed coronal structures (e.g. Aulanier et al. 2000) driven unstable by foot point shearing and/or flux emergence or cancellation.

\subsection{Simple CME model applied here}

In the present study, we are primarily interested in the evolution of the MHD shocks generated by CMEs in the solar wind rather than in the evolution of the three-part CME structure itself. Therefore, we used the simple "density(+pressure)driven" model that is superposed on the three different wind models. Hence, in our simulations the shocks are generated by launching a high-density and high-pressure plasma "blob" on the wind at a certain velocity $v_{\text {cme }}$ in a prescribed radial direction $\theta_{\text {cme }}$. The velocity and density profile in the initial disturbance are both of the form:

$y=\frac{y_{\mathrm{cme}}}{2}\left(1-\cos \pi \frac{d_{\mathrm{cme}}-d}{d_{\mathrm{cme}}}\right)$,

where $y$ indicates the density $\rho$ or radial velocity $v_{r}, y_{\text {cme }}$ is the maximum density or radial velocity in the plasma bubble, 


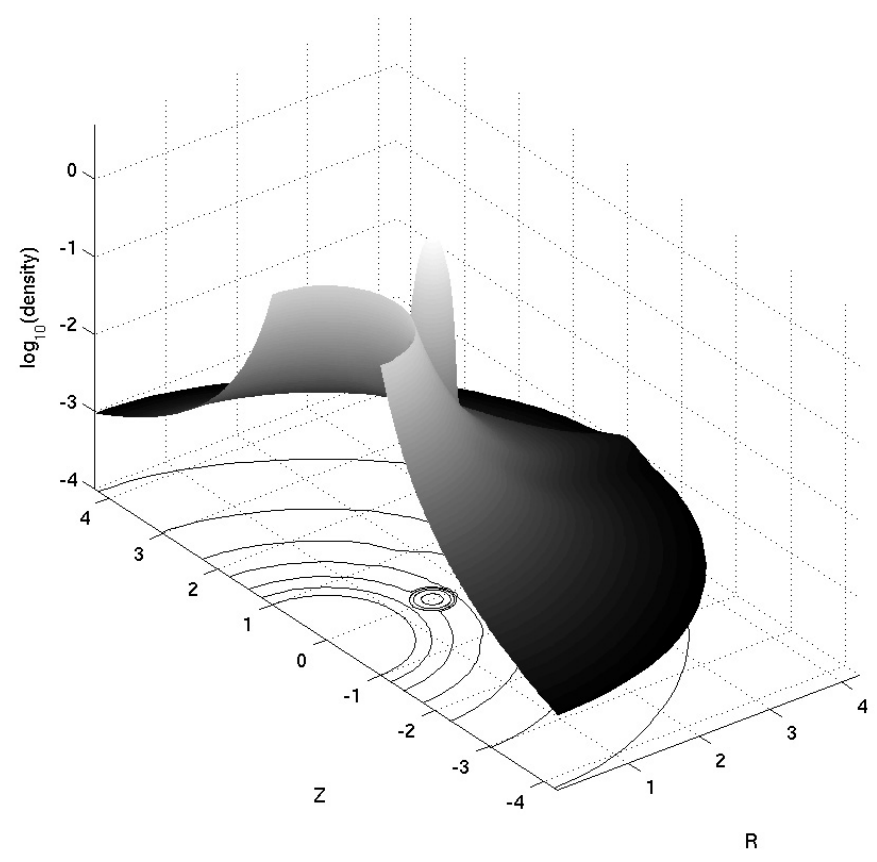

Fig. 3. The initial (normalized) density profile used for the shock generation. Model 1 background wind.

$d_{\text {cme }}$ is the radius of the bubble and $d$ the distance to the center of the bubble. By choosing the profile like this, there will be a smooth transition between the perturbed region and the background wind. The center of the initially superposed plasma blob is at a certain radial distance $r_{\mathrm{cme}}$ and latitude $\theta_{\mathrm{cme}}$. For all simulations shown in this article $r_{\text {cme }}$ has a value of $1.5 R_{\odot}, \theta_{\text {cme }}$ equals a latitude of $0^{\circ}$ or $60^{\circ}$ and $d_{\text {cme }}$ equals $0.29 R_{\odot}$. In Fig. 3 a surface plot is shown of the initial density profile for the simulation in the equatorial plane $\left(\theta_{\mathrm{cme}}=0^{\circ}\right)$.

For every simulation discussed in this paper the maximum density $\rho_{\text {cme }}$ in the bubble has a value of $5 N_{*}$, i.e. five times the density at the reference position, and the peak radial velocity $v_{\text {cme }}$ is about $1000 \mathrm{~km} \mathrm{~s}^{-1}$, in order to create a fast MHD shock. For the polytropic wind models the extra enhancement in pressure is directly related to the density profile by the polytropic relation $p=\rho^{\gamma} / \gamma$. The extra pressure added to wind model 2 is taken exactly equal to the pressure in the disrupting plasma bubble on top of the polytropic wind in order to have exactly the same initial disturbance superposed on each of the three different winds.

For reasons of numerical stability, back flow is not allowed through the boundary: if $v_{r}<0$ than $v_{r}$ is put equal to zero on the boundary. Hence, we do not allow that flow is going back into the sun, which does not mean that there cannot be back flow in the simulation domain. Except from this, the boundary conditions used for these simulations are the same as to generate the background wind, and so the wind re-achieves its original steady state configuration when the simulation is ran for a long enough time.

\section{CME propagation}

In Fig. 4 the results of the simulations are visualized, $4 \mathrm{~h} 12 \mathrm{~min}$ after onset. Snapshots of the total (i.e. wind + CME) density, the relative density, defined as

$\bar{\rho}=\left(\rho_{t}-\rho_{w}\right) / \rho_{w}$,

the total radial velocity, and difference between the total radial velocity and the radial velocity of the background wind, i.e.

$\Delta v_{r}=v_{r_{t}}-v_{r_{w}}$,

are shown, where the subscript $t$ and $w$ denotes respectively total and background wind. From this one can see that the density and velocity profile of the wind influence the evolution of the shock. In the model 2 wind there are strong density and velocity gradients in the north-south direction because of the extra heating term, leading to a blobbed shape for the shock when $\theta_{\text {cme }}=0^{\circ}$. This in contrast with the two polytropic wind models, which have much smoother gradients in density and velocity, what gives an oval shape to the shock front. In Fig. 5 the profiles of density, velocity and temperature are shown along a cut in the launching direction ( $4 \mathrm{~h} 12 \mathrm{~min}$ after onset). This demonstrates not only the differences between the winds (dashed lines), but also the differences in behaviour of the shock propagation. Because the density and velocity in the model 1 wind is lower than in the other winds, the impact of the disturbance is higher and steeper shocks are generated.

The effect of the background wind on the propagation speed of the leading shock is clearly visible when a time sequence for the position of the shock front is plotted (Fig. 6). Due to the faster polar wind profile of model 2, the disturbance will propagate faster in this model, which is particularly clear when launched on $\theta_{\mathrm{cme}}=60^{\circ}$. For the simulation on $\theta_{\mathrm{cme}}=0^{\circ}$, the higher density in the equatorial plane for the model 2 and model 3 wind, compared to model 1, acts like an obstacle making the CME to move slower, although the background wind velocity is slightly higher for these two wind models. Heighttime plots for the position of the shock front in the direction of the launch angle are shown in Fig. 7. A linear fit of the ridges in the height-time plots gives a value for the average propagation speed of the shock front (in the launching direction). The average propagation speeds are summarized in Table 2 .

Looking at Figs. 4a and $6 a$ one can see a small dimple in the shockfront at the position of the equator. However, this dimple is not of the same origin as the dimpled shocks discussed in De Sterck (1999). Dimpled shocks with their complex topology can only occur in a certain flow regime which is called "magnetically dominated flow". In the case of our simulations, it turns out that the flow is not magnetically dominated, but instead pressure dominated. This is not suprising, because in our simulations no extra magnetic field is included and the background field is weak. The dimple in the shock front on the equator is purely caused by density effects. All three wind models have an increased density in the equatorial region (see Fig. 2), creating a density gradient which slows down the CME in the equatorial plane. As a preliminary result we can say that the shocks away from the equator created in our simulations are of the fast type. In a fast shock the magnetic field is bent away 


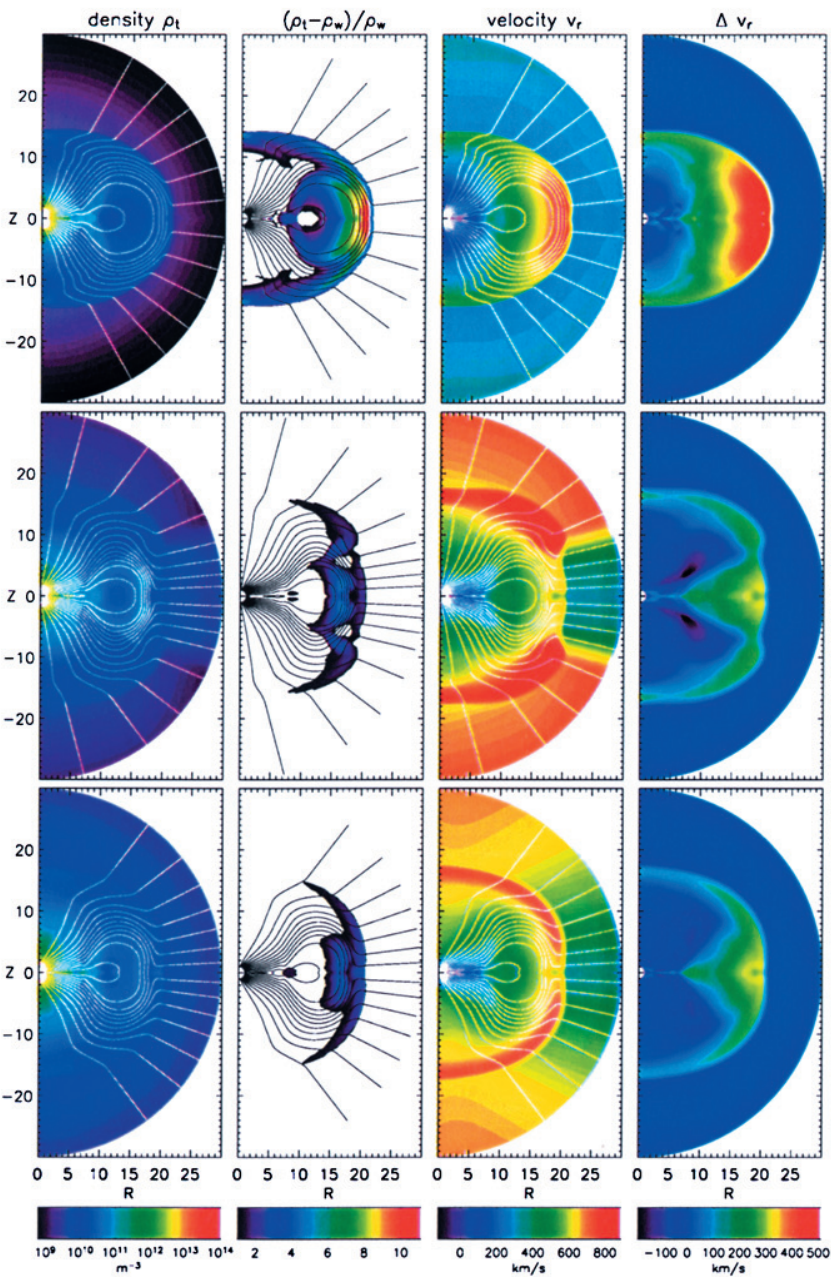

(a) $\theta_{\mathrm{cme}}=0^{\circ}$

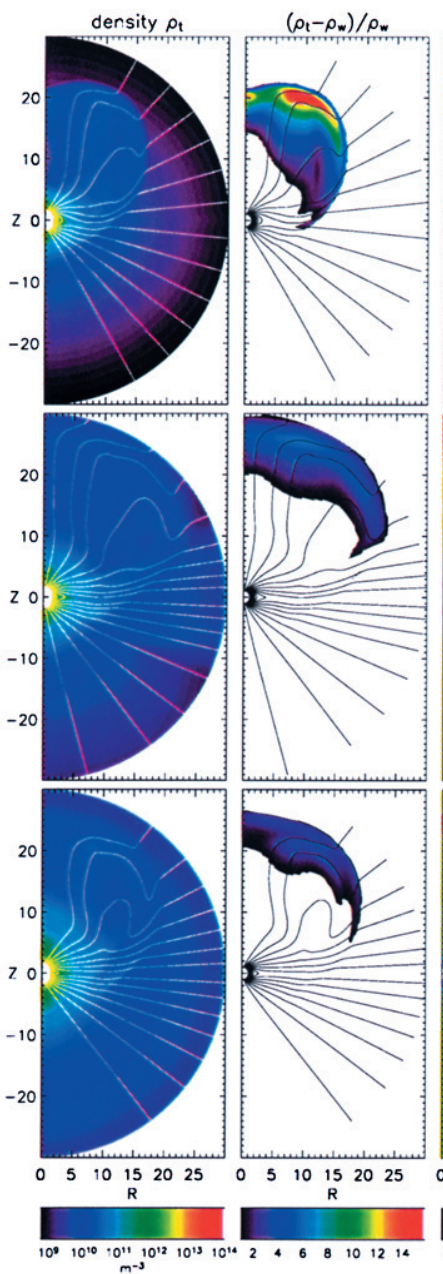

(b) $\theta_{\mathrm{cme}}=60^{\circ}$

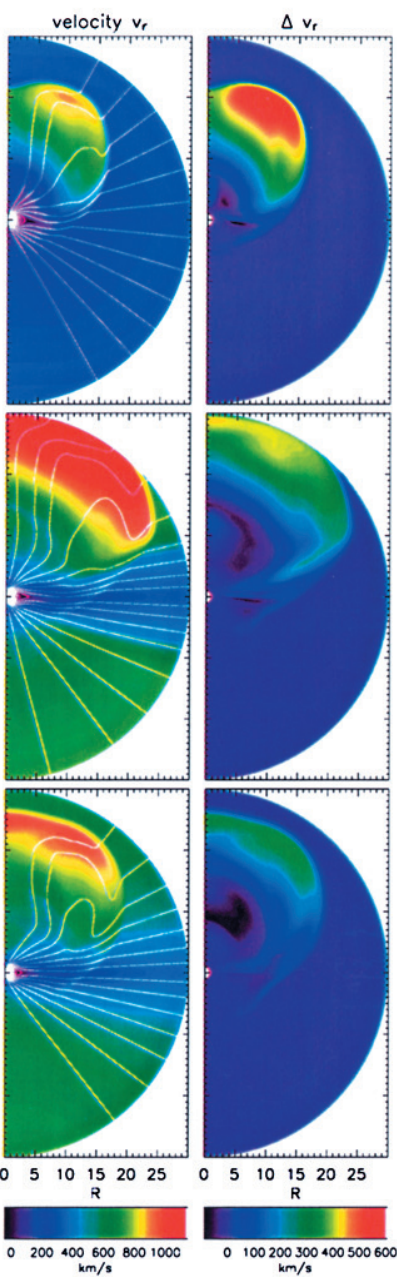

Fig. 4. Snapshots of (from left to right) total density profile, relative density, radial velocity and difference in radial velocity with the background wind for (a) $\theta_{\mathrm{cme}}=0^{\circ}$ and for (b) $\theta_{\mathrm{cme}}=60^{\circ}$. Magnetic field lines are shown in black or white. For clearness, the contour lines (dotted) of the relative density are shown. Above: model 1 wind; middle: model 2 wind; below: model 3 wind. (This figure is available in color in electronic form.)

from the shock normal in the region downstream of the shock. Looking at a plot of the density and magnetic field on top of each other (Fig. 8) one would indeed expect to find a fast shock.

When launching the CME along the equator, the magnetic field of the background wind plays an important role in the evolution of the plasma blob. In this case the initial perturbation is completely inside the closed magnetic field lines and magnetic tension forces slow down the speed of the plasma blob. This in contrast to the case $\theta_{\mathrm{cme}}=60^{\circ}$ : now the bubble is initially completely outside the closed field line region and during the evolution of the plasma blob the magnetic field plays a minor role in the propagation. The pressure-density disturbance deforms the magnetic field lines and pushes the helmet streamer, which bends away as is observed during a CME event (Sheely et al. 2000). Launching the plasma blob outside the closed magnetic field lines may not seem to be realistic in the study of CME propagation. The reason to do so, however, is to verify the effect on the magnetic field, and to see how the wind velocity affects the propagation of the plasma blob.
The simulations demonstrate that the chosen wind model does not only influence the propagation speed, but also the spread angle of the CME: two quantities that are of importance if one wants to determine the geo-effectiveness of a CME event. Because these are only 2.5D simulations, defining a spread angle only makes sense for the simulations in the equatorial plane. To quantify the spread angle the relative density is used. The mass released in a CME event is quite substantial and the relative density is a tool to point out the position of the plasma blob in our simulations. The mass inside the blob is called relevant when the total density is at least two times higher than the steady state wind density. This is exactly what is shown in the second column of Figs. $4 \mathrm{a}$ and $4 \mathrm{~b}$. By this, the spread angle of the plasma blob can be determined. A visualization of the evolution of the spread angle in time is given in Fig. 9. In the model 1 background wind the shock immediately spreads out over $180^{\circ}$, while for the other models it stays more confined in space. The explanation for this effect is the lower density profile in wind model 1 and so the higher impact of the disturbance. 


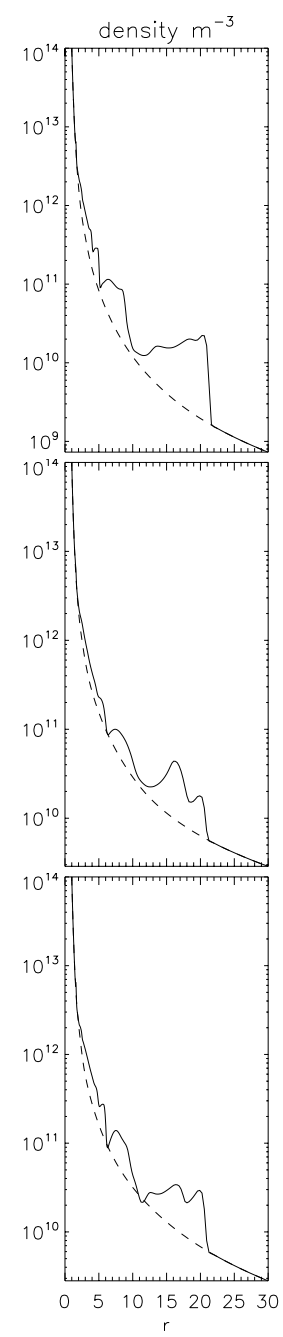

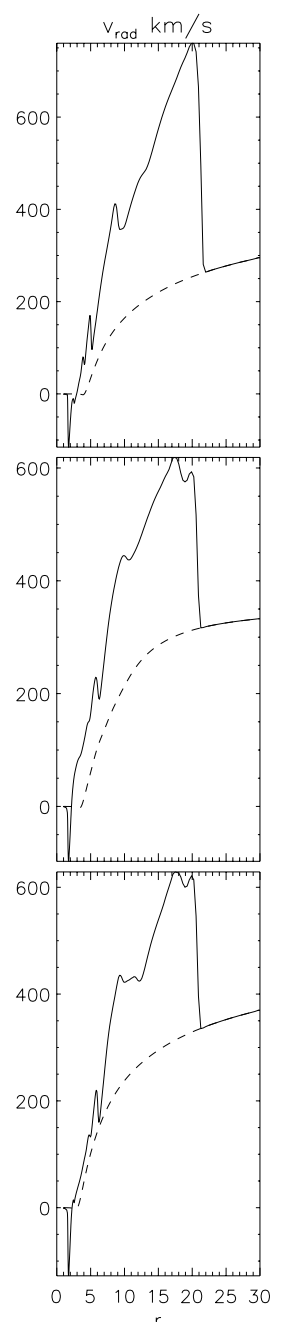

(a) $\theta_{\mathrm{cme}}=0^{\circ}$
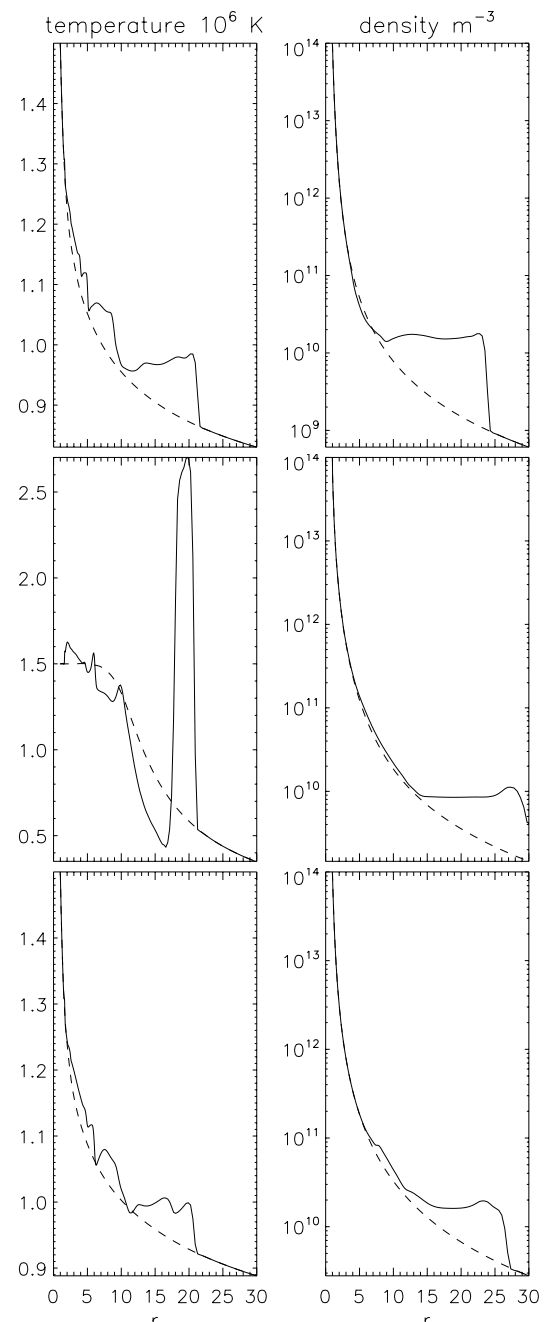

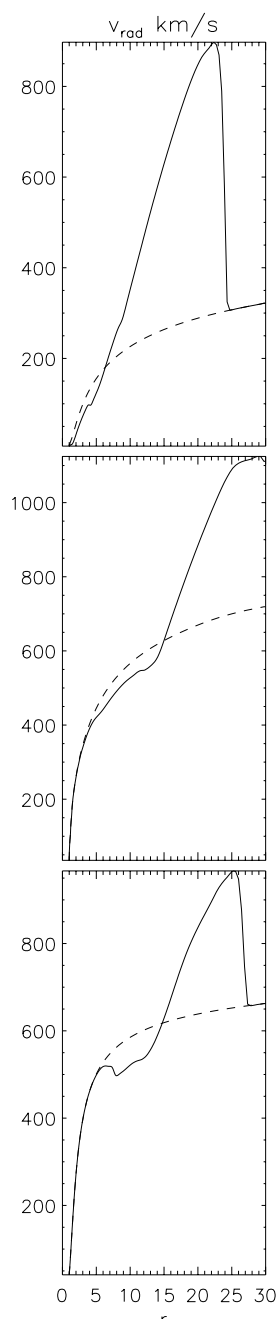

(b) $\theta_{\mathrm{cme}}=60^{\circ}$

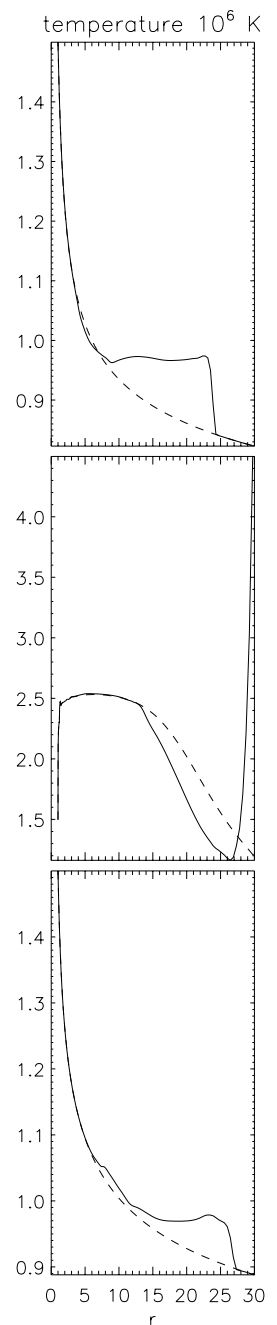

Fig. 5. Cuts along the launching direction of (from left to right) density, radial velocity and temperature for (a) $\theta_{\mathrm{cme}}=0^{\circ}$ and for (b) $\theta_{\mathrm{cme}}=60^{\circ}$. The dashed line is the profile of the background wind. Above: model 1 wind; middle: model 2 wind; below: model 3 wind.

\section{Current sheet formation and reconnection effects}

Let us concentrate on the simulation with $\theta_{\text {cme }}=0^{\circ}$. In the plots of the relative density (Fig. 4a) a small enhancement in density is seen around $10 R_{\odot}$, lagging behind the plasma blob for all three wind models. This feature is better visible in Fig. 5a and at the same position also an increase of velocity and temperature (the latter clearly present in the second wind model) is located. The same features are visible at about $5 R_{\odot}$. What is the explanation for this increase in density and velocity? Because of the eruption, the closed field lines of the helmet streamer are distorted and stretched out. Behind the ejected plasma blob, the field lines are approaching each other, creating a current sheet and a X-type magnetic structure in the equatorial plane (see Fig. 10). Although the simulations were performed with an ideal MHD code, numerical dissipation/resistivity cannot be excluded, and this has the same effect as including real magnetic resistivity. Reconnection processes detach the upper part of the helmet streamer from the solar surface, giving rise to an outward moving flux rope and a newly formed helmet streamer. Strong vertical components for the velocity are developing just above and under the reconnection position, indicating the existence of flow in the direction of the reconnection site. As the magnetic field lines approach each other, the density in between rises, the plasma is heated and is finally squeezed out when the reconnection occurs, giving massflows towards and away from the sun (see also Riley et al. 2002), which is visualized in Fig. 10 and explains the features in Fig. 5a. The back flows in our simulations can have velocities up to $200 \mathrm{~km} \mathrm{~s}^{-1}$. Several authors report on observations of such a back flows (e.g. McKenzie \& Hudson 1999; Asai et al. 2004) and find speeds of 100-200 $\mathrm{km} \mathrm{s}^{-1}$ (McKenzie $\&$ Hudson 1999). Although the simulation results are in agreement with observations, we should keep in mind that the back flow generated in our simulation is a purely numerical effect.

Figure 10 shows the magnetic field lines, velocity vectors and a contour plot of the azimuthal component of the current density among the reconnection site $7 \mathrm{~h} 48 \mathrm{~min}$ after the onset of the eruption, this is right after when the shock has moved completely outside the simulation domain. During the 

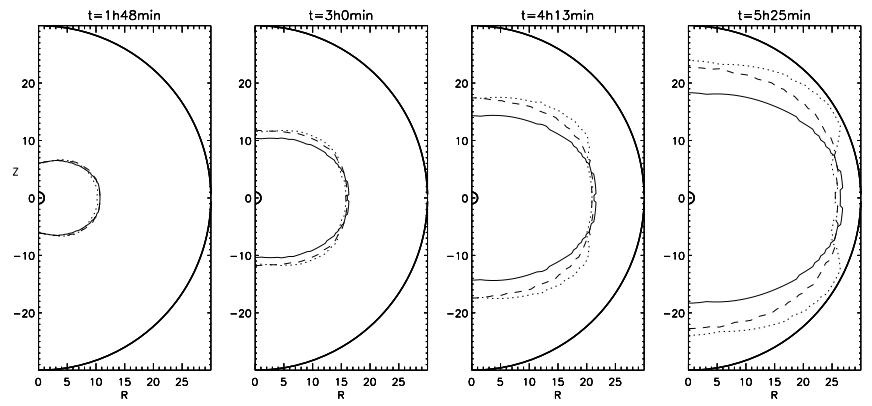

(a) $\theta_{\mathrm{cme}}=0^{\circ}$
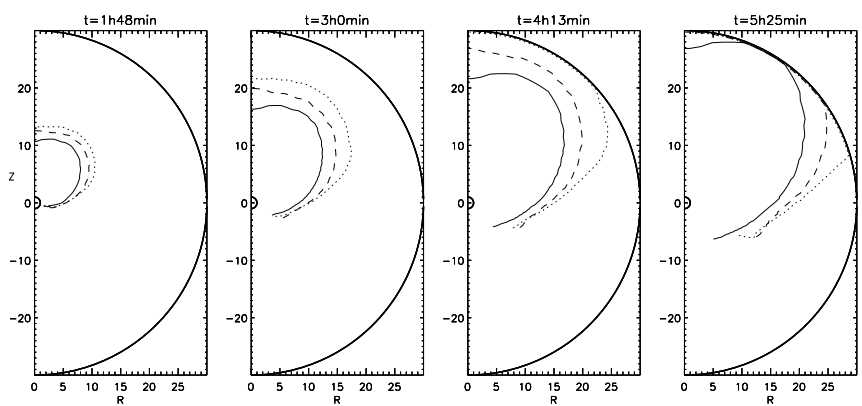

(b) $\theta_{\mathrm{cme}}=60^{\circ}$

Fig. 6. Position of the shock front for different times. Solid line: model 1 wind; dotted line: model 2 wind; dashed line: model 3 wind. The thick solid line indicates the boundaries of the simulation domain.

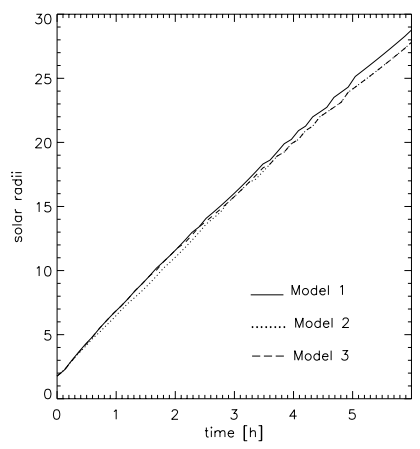

(a) $\theta_{\text {cme }}=0^{\circ}$

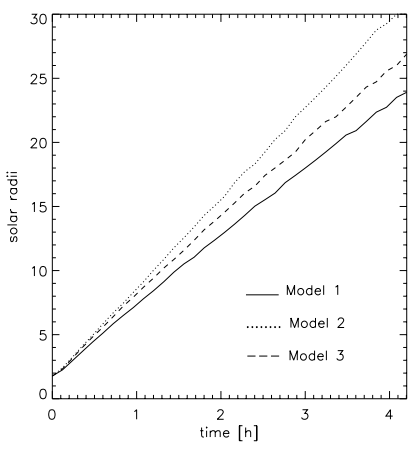

(b) $\theta_{\mathrm{cme}}=60^{\circ}$
Fig. 7. Position of the shock front versus time, in the direction of the launch angle. Solid line: model 1 wind; dotted line: model 2 wind; dashed line: model 3 wind.

Table 2. Average propagation speed of the shock front along the launch angle.

\begin{tabular}{llll}
\hline \hline & Model 1 & Model 2 & Model 3 \\
\hline$\theta_{\mathrm{cme}}=0^{\circ}$ & $865 \mathrm{~km} \mathrm{~s}^{-1}$ & $845 \mathrm{~km} \mathrm{~s}^{-1}$ & $835 \mathrm{~km} \mathrm{~s}^{-1}$ \\
$\theta_{\mathrm{cme}}=60^{\circ}$ & $1030 \mathrm{~km} \mathrm{~s}^{-1}$ & $1355 \mathrm{~km} \mathrm{~s}^{-1}$ & $1160 \mathrm{~km} \mathrm{~s}^{-1}$ \\
\hline
\end{tabular}

simulation the helmet streamer slowly regains its original structure, in agreement with reports of observational studies (Švestka et al. 1995), the back flows become less and less strong, and approximately $50 \mathrm{~h}$ after the start of the simulation the wind is again in its original steady state.
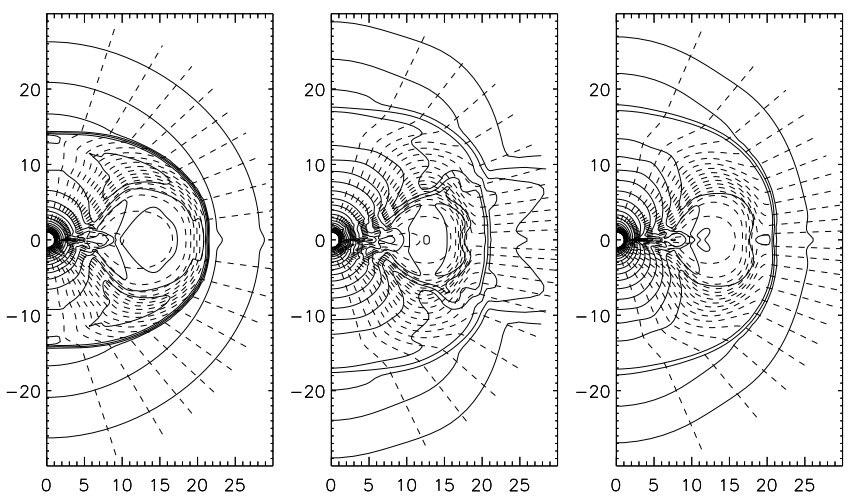

Fig. 8. Contour lines of density (solid), overplotted with magnetic field lines (dashed). The upstream region is the area behind the shock, away from the sun.

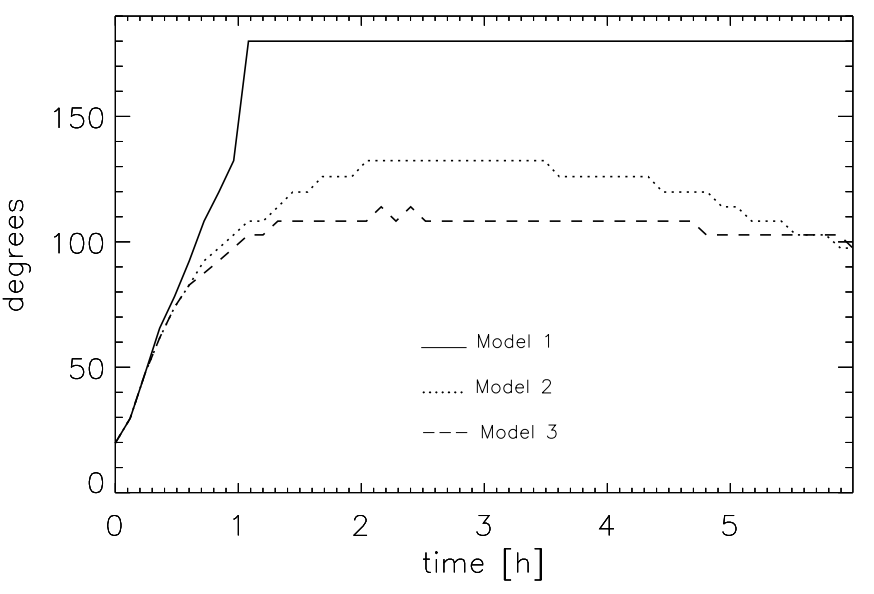

Fig. 9. Evolution of the spread angle of the plasma blob in time. Solid line: model 1 wind; dotted line: model 2 wind; dashed line: model 3 wind.

\section{Conclusions and future plans}

For the solar wind there exist many advanced 1D, 2D, and 3D steady state models with state-of-the-art numerical techniques and/or a lot of physical effects included. Yet, these models are all steady state models which are still far away from a realistic, high-resolution, real-time unsteady model. Moreover, the present wind models use a lot of additional (artificial) heating and/or momentum source terms which demonstrate and circumvent our lack of physical insight. Also, there are only a few attempts so far to include non-MHD effects, at least in the solar wind models used for CME evolution studies.

In spite of these drawbacks, the simplified 2.5D solar wind models presented here demonstrate the importance of an accurate and reliable wind model in the framework of space weather predictions. By reconstructing three popular wind models, on exactly the same grid, with the same numerics and same boundary conditions, we could demonstrate that the use of different models for the pre-event solar atmosphere leads to substantially different behavior in CME propagation and evolution. The simulations presented here were only performed up to $30 R_{\odot}$ and the CME initiation model used was rather simplistic. Nevertheless, differences in shock speed, shock strength, 


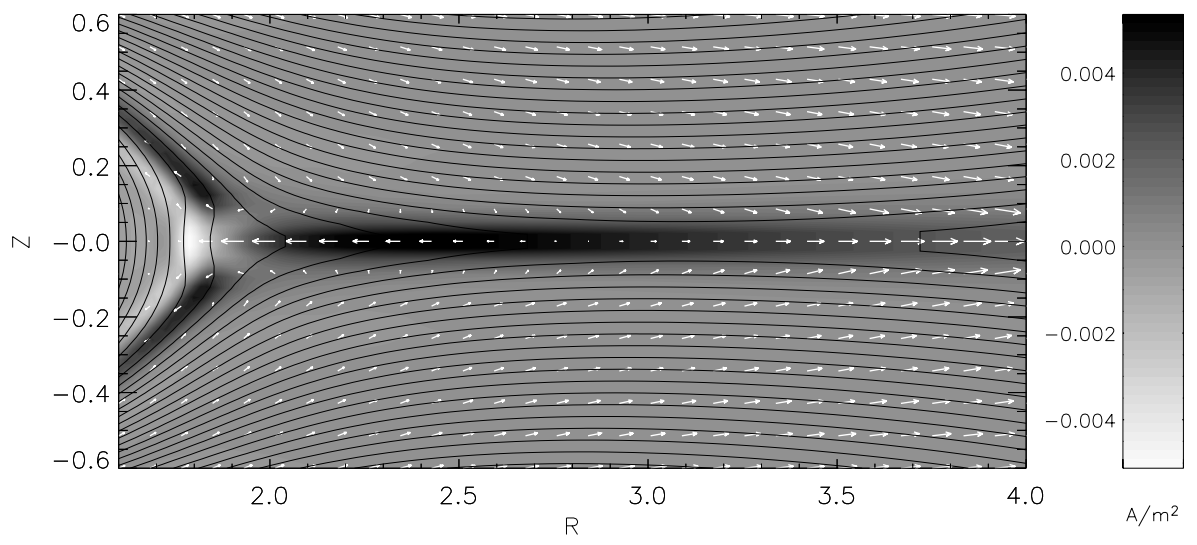

Fig. 10. Contour plot of the current density $\left(J_{\phi}\right)$, overplotted with magnetic field lines (black) and velocity vectors (white), at the position of the reconnection site. Background wind model: polytropic with Alfvén waves. spreadangle and mass distribution can be found and quantified. It would be interesting to see how these disturbances propagate up to $1 \mathrm{AU}$, and how the background wind model influences the time of arrival, the spreadangle and the mass-impact of a CME-event. This will be investigated in the near future.

Another important factor in space weather is the magnetic field inside a magnetic cloud, caused by a CME-event, approaching the earth. In a later paper we will investigate the effect of the magnetic polarity in the CME flux rope. We will show that the orientation of the flux rope magnetic field, with respect to the background field, significantly influences the evolution path of the CME.

As mentioned in the introduction, the shocks generated in MHD can have a complex topology and can reveal some information about the background plasma. The investigation of the propagating shock topology and evolution of the shock topology in space and time is rather complicated and further analysis is still going on. The results obtained thus far, tell us that the flow is pressure dominated and that away from the equator the shocks are of the fast type.

Of course these simulations are only a beginning and a simplification of a very complex topic. More realistic (3D) CME models, real time predictions of the solar wind conditions, and an extension of the simulations to at least $1 \mathrm{AU}$ are a necessary next step.

Acknowledgements. These results were obtained in the framework of the projects OT/02/57 (K.U. Leuven), 14815/00/NL/SFe(IC) (ESA Prodex 6), and the European Community's Human Potential Programme contract HPRN-CT-2000-00153, PLATON, also acknowledged by B. vdH.

\section{References}

Asai, A., Yokoyama, T., Shimojo, M., \& Shibata, K. 2004, ApJ, 605, L77

Aulanier, G., DeLuca, E. E., Antiochos, S. K., et al. 2000, ApJ, 540, 1126

Burlaga, L. F., Plunkett, S. P., \& St. Cyr, O. C. 2002, J. Geophys. Res., 107, SSH 1

De Sterck, H., \& Poedts, S. 1999, A\&A, 343, 641

De Sterck, H., Low, B. C., \& Poedts, S. 1999, Phys. Plasmas 6 (3), 954

Gibson, S., \& Low, B. C. 1998, ApJ, 493, 460
Gombosi, T. I., DeZeeuw, D. L., Groth, C. P. T., Powell, K. G., \& Stout, Q. F. 2000, J Atm. and S-T Phys., 62, 1515

Gopalswamy, N., Yashiro, S., Kaiser, M. L., Howard, R. A., \& Bougeret, J.-L. 2001, ApJ, 548, L91

Groth, C. P. T., De Zeeuw, D. L., Gombosi, T. I., \& Powell, K. G. 2000, J. Geophys. Res., 105, 25053

Hollweg, J. V. 1973, ApJ, 181, 547

Jacques, S. A. 1977, ApJ, 215, 942

Keppens, R., \& Goedbloed, J. P. 1999, A\&A, 343, 251

Keppens, R., \& Goedbloed, J. P. 2000, ApJ, 530, 1036

Klimchuk, J. A. 2001, Proc. of the Chapman Conference on Space Weather, AGU, Geophysical Monograph Series, 125, ed. P. Song, H. Singer, \& G. Siscoe, 143

McKenzie, D. E., \& Hudson, H. S. 1999, ApJ, 519, L93

Odstrčil, D., \& Pizzo, V. J. 1999, J. Geophys. Res., 104, 483 and 493

Pneuman, G. W., \& Kopp, R. A. 1971, Sol. Phys., 18, 258

Poedts, S., Van der Holst, B., Chattopadhyay, I., et al. 2003, Proc. ISCS 2003 Symp., Solar Variability as an Input to the Earth's Environment, Tatranská Lomnica, Slovakia, 23-28 June 2003, ESA SP-535, 603

Riley, P., Linker, J. A., Mikić, Z., et al. 2002, ApJ, 578, 972

Roussev, I. I., Forbes, T. G., Gombosi, T. I., et al. 2003, ApJ, 588, L45

Sakurai, T. 1985, A\&A, 152, 121

Sakurai, T. 1990, Comput. Phys. Rep., 12, 247

Sheeley, N. R., Hakala, W. N., \& Wang, Y.-M. 2000, J. Geophys. Res., 105,5081

Steinolfson, R. S., \& Hundhausen, A. J. 1990, J. Geophys. Res., 95, 20693

Suess, S. T., Wang, A. H., Wu, S. T., Poletto, G., \& McComas, D. J. 1999, J. Geophys. Res., 104, 4697

Švestka, Z., František, F., Hudson, H. S., et al. 1995, Sol. Phys., 161, 331

Terasawa, T., Kawada, S., Ipavich, F. M., et al. 2002, Proc. First Stereo Workshop, Paris, in press

Titov, V. S., \& Démoulin, P. 1999, A\&A, 351, 701

Tóth, G. 1996, Astrophys. Lett. \& Comm., 34, 245

van der Holst, B., Van Driel-Gesztelyi, L., \& Poedts, S. 2002a, ESA SP-506, 71

van der Holst, B., Banerjee, D., Keppens, R., \& Poedts, S. 2002b, Proc. 10th European Solar Physics Meeting (SPM-10), Solar variability: from core to outer frontiers, 9-14 September 2002, Prague, Czech Republic, ESA SP-506, 75

Wang, A. H., Wu, S. T., Suess, S. T., \& Poletto, G. 1995, Sol. Phys., 161,365

Wang, A. H., Wu, S. T., Suess, S. T., \& Poletto, G. 1998, J. Geophys. Res., 103, 1913

Weber, E. J., \& Davis, L., Jr. 1967, ApJ, 148, 217 